\title{
Nondifferentiable optimization solver: basic theoretical assumptions
}

\author{
Andrzej Stachurski \\ Institute of Automatic Control, Warsaw University of Technology \\ Nowowiejska 16/19, Warszawa, Poland. Tel: \& Fax: +48 22-253719. \\ e-mail: stachurski@ia.pw.edu.pl
}

\begin{abstract}
The paper presents algorithms realized in NDOPT - the code designed for solving nondifferentiable convex optimization problems. The main algorithm is based on the use of the exact penalty function and linearizations of the nonlinear constraints. Its novelty lies in the original development of the solution method of the QP direction search problem. A strictly convex reduced primal direction search problem is solved, not dual as usual.
\end{abstract}

\section{Keywords}

nondifferentiable convex optimization, exact penalty function, linearization, primal direction search problem

\section{INTRODUCTION}

NDOPT is a part of the DIDASN ++ package designed for multicriteria optimization and modelling. It is one of the optimization solvers included in DIDASN ++ . The aim of this paper is to present the algorithms realized in this code.

NDOPT is a collection of standard ANSI C functions designed to solve small-scale nondifferentiable optimization problems of the following standard form

minimize $f(x)=\max \left\{f_{j}(x): \quad j=1, \ldots, m_{O}\right\}$,

subject to

$y_{j}^{L} \leq F_{j}(x) \leq y_{j}^{U}, \quad$ for $\quad j=1, \ldots, m$,

$b^{L} \leq A x \leq b^{U}$

$x_{i}^{L} \leq x_{i} \leq x_{i}^{U} \quad$ for $\quad i=1, \ldots, n$,

Sponsored by KBN, grant No. 3 P40301806 "Parallel computations in linear and nonlinear optimization" 
where the vector $x=\left(x_{1}, \ldots, x_{n}\right)^{T}$ has $n$ components, $f_{j}$ and $F_{j}$ are locally Lipschitz continuous functions, $A$ is an $m_{L} \times n$ matrix, $y^{L}$ and $y^{U}$ are constant $m$-vectors, $b^{L}$ and $b^{U}$ are constant $m_{L}$-vectors and $x^{L}$ and $x^{U}$ are constant $n$-vectors. Matrix $A$ is assumed to be dense.

The nonlinear functions $f_{j}$ and $F_{j}$ have to be Lipschitz continuous, however they need not be continuously differentiable (have continuous gradients, i.e. vectors of partial derivatives). In particular, they may be convex. The user has to provide a $\mathrm{C}$ function for evaluating the problem functions and their single subgradients (called generalized gradients) at each $x$ satisfying the linear constraints. For example, if $F_{j}$ is continuously differentiable then its subgradient $g_{F_{j}}(x)$ is equal to the gradient $\nabla F_{j}(x)$ and if $F_{j}$ is a max function

$F_{j}(x)=\max \{\varphi(x, z) ; \quad z \in Z\}$

i.e. $F_{j}(x)$ is a pointwise maximum of smooth functions and $Z$ is compact, then $g_{F_{j}}(x)$ may be calculated as the gradient $\max _{z \in Z\langle x)} \partial_{x} \varphi(x, z)$ (with respect to $x$ ), where $Z(x)$ is an arbitrary solution to the maximization problem in (5). (Surveys of subgradient calculus, which generalizes rules like $\partial\left(F_{1}+F_{2}\right)(x)=\partial F_{1}(x)+\partial F_{2}(x)$, may be found in Clarke (1983).

NDOPT implements the descent methods applied to the exact penalty function for problem (1-4). That approach have been recently intensively studied by Kiwiel in numerous papers, e.g. Kiwiel $(1985,1988)$.

\section{EXACT PENALTY FUNCTION}

We assume the use of the exact penalty function with respect to the nonlinear constraints. Problem (1-4) is therefore internally transformed to the following condensed form:

minimize $f(x)$ over all $x \in R$

subject to

$F(x) \leq 0$,

$b^{L} \leq A x \leq b^{U}$

$x^{L} \leq x \leq x^{U}$,

where $f$ is the objective function, $F$ is the aggregate constraint function

$F(x)=\max \left(F^{L}, F^{U}\right), \quad$ where

$F^{L}=\max \left\{y_{j}^{L}-F_{j}(x): j=1, \ldots, m\right\}$ - aggregate lower inequality constraint function, $F^{U}=\max \left\{F_{j}(x)-y_{j}^{U}: j=1, \ldots, m\right\}$ - aggregate upper inequality constraint function.

The $m_{L}$ inequalities (8) are called the general linear constraints, whereas the box constraints (9) specify upper and lower simple bounds on all variables. 
The standard form (1-4) is more convenient to the user than (6-9), since the user does not have to program additional operations for evaluating the functions $F^{L}$ and $F^{U}$ and their subgradients. On the other hand, the condensed form simplifies the description of algorithms.

Let $S_{L}$ denotes the feasible set defined by the linear constraints

$S_{L}=\left\{x \in R^{n} ; b_{L} \leq A x \leq b^{U}, x^{L} \leq x^{U}\right\}$

and let's define the exact penalty function with the penalty coefficient $c>0$

$e(x ; e)=f(x)+c \max \{F(x), 0\}$.

Given a fixed $c>0$, any solution $x_{e}$ to the problem

$\min e(x ; c) \quad$ over all $\quad x \in S_{L}$

solves problem $(6-9)$, if $x_{e}$ is feasible, i.e. $\left(F\left(x_{e}\right) \leq 0\right)$. This holds, if the penalty parameter is sufficiently large, problem $(6-9)$ has a solution and its constraints are sufficiently regular.

\section{THE LINEARIZATION ALGORITHM}

We assume that NDOPT algorithms has to generate points feasible with respect to the linear constraints, i.e. consecutive approximate solutions to problem $(6-9)$ should lie within $S_{L}$. The user has to specify an initial estimate $x^{0}$ of the optimal solution and its orthogonal projection on $S_{L}$ is taken as the algorithm's starting point $x^{1}$.

The algorithms in NDOPT are based on the concept of descent methods for nondifferentiable minimization. Starting from a given approximation to a solution of (1-4), an iterative method of descent generates a sequence of points, which should converge to a solution. The property of descent means that successive points have lower objective (or exact penalty) function values. To generate a descent direction from the current iterate, the method replaces the problem functions with their piecewise linear (polyhedral) approximations. Each linear piece of such approximation is a linearization of the given function, obtained by evaluating the function and its subgradient at a trial point of an earlier iteration. (This generalizes the classical concept of using gradients to linearize smooth functions.) The polyhedral approximations and quadratic regularization are used to derive a local approximation to the original optimization problem, whose solution (found by quadratic programming) yields the search direction. Next, a line search along this direction produces the next approximation to a solution and the next trial point, detecting the possible gradient discontinuities. The successive approximations are formed to ensure convergence to a solution without storing too many linearizations. Rejecting the oldest subgradients from the bundle of those collected from the beginning of the computational process one avoids the potential problems with the lack of computer memory.

Each step of the algorithm consists of the following three main parts:

- generation a consecutive piecewise linear approximation of all nonlinear functions 
- solution of the direction finding subproblem

- directional minimization to either find a new approximate solution with a smaller objective function value or deduce that the current search direction is not the descent direction. In both cases we get a new trial point which gives information necessary to generate a new linear approximation.

The first part is obvious. In the second an original primal approach is proposed. This is in contrary to the usual dual approach. In the third we shall investigate an approach based on the use of two linear supporting functions with opposite derivative signs. The last property ensures the existence of the intersection point inside the interval of the two trial points. These topics are discussed in next sections. Formulation of the main algorithm follows below:

Step 0 (奋itialization). Choose a starting point $x^{1} \in S_{L}$, final accuracy tolerance $\epsilon_{s} \geq 0$, direction search parameter $m \in(0,1)$, maximal number $m_{g}$ of stored subgradients of $e(x ; c)$, starting value $c^{1}>0$ of the penalty parameter and the starting value of the accuracy tolerance $\delta^{1}>0$ of the penalty function minimization. Set $y^{1}=x^{1}$, $J_{e}^{1}=\{1\}, e_{1}^{1}=e\left(y^{1} ; c^{1}\right), g_{e}^{1}\left(y^{1} ; c^{1}\right), k=1$.

Step 1 (Direction finding) Find the optimal solution $\left(d^{k}, v^{k}\right)$ of the QP direction search problem:

$$
\begin{array}{r}
\min \frac{1}{2}|d|^{2}+v \quad \text { with respect to }(d, v) \in R^{N+1} \\
e_{j}^{k}+<g_{e}^{k, j}, d>\leq v \\
x^{k}+d \in S_{L}
\end{array} \quad \quad j \in J_{e}^{K},
$$

and the Lagrange multipliers $\lambda$ - corresponding to the inequalities following from the hyperplanes supporting the epigraph of the penalty function from below, $\nu$ corresponding to general linear constraints and $\mu$-corresponding to box constraints respectively. Let $\hat{J}_{e}^{k} \in J_{e}^{k}$ denotes the subset of the indices of the first group of constraints with positive multipliers $\left(\lambda_{j}>0, j \in \hat{J}_{j}^{k}\right)$. Compute $\left.\left.V^{k}=v^{k}-e\right) x^{k} ; c^{k}\right)$.

Step 2 (Stopping criterion) If $V^{k} \geq-\epsilon^{s}$ and $F\left(x^{k}\right) \leq \epsilon_{s}$ then STOP else continue.

Step 3 (Penalty coefficient modification) If $\left|V^{k}\right| \leq \delta^{k}$ and $F\left(x^{k}\right)>\left|V^{k}\right|$ then set $c^{k+1}=$ $2 * c^{k}$ and $\delta^{k+1}=\delta^{k} / 2$ and recalculate respectively values of stored subgradients of the penalty function $g_{j}^{k} ; j \in J_{e}^{k}$; otherwise set $c^{k+1}=c^{k}$ and $\delta^{k+1}=\delta^{k}$.

Step 4 (Direction search) Set $y^{k+1}=x^{k}+d^{k}$. If

$$
e\left(y^{k+1} ; c^{k+1}\right) \leq e\left(x^{k} ; c^{k+1}\right)+m * V^{k},
$$

then set $x^{k+1}=y^{k+1}$ (serious step); otherwise set $x^{k+1}=x^{k}$ (zero step). Compute $g_{e}^{k+1}$.

Step 5 (Modification of linearizations) Choose a subset of $\tilde{J}_{e}^{k} \subset J_{e}^{k}$ satisfying $\tilde{J}_{e}^{k} \supset \hat{J}_{e}^{k}$ and $\left|\tilde{J}_{e}^{k}\right| \leq M_{g}-1$. Augment $\tilde{J}_{e}^{k}$ with the newest linear approximation of the penalty function $e\left(y^{k+1} ; c^{k+1}\right)$ setting $J_{e}^{k+1}=\tilde{J}_{e}^{k}+k+1$ and recalculate values of linearizations $e_{j}^{k+1}, j \in J_{e}^{k+1}$, if necessary. Increase $k$ by 1 and return to Step 1 . 


\section{AUXILIARY DIRECTION SEARCH QP PROBLEM}

The direction finding subproblem has got the form of a following convex (unfortunately not strictly convex) QP programming problem:

$$
\min _{(d, v) \in R^{n} \times R^{1}} \frac{1}{2}|d|^{2}+v
$$

subject to

$$
\begin{aligned}
& -\alpha_{i}+P_{i}^{T} d \leq v, \text { where } i=1,2, \ldots, m, \\
& y^{L} \leq A^{T}\left(x^{k}+d\right) \leq y^{U} \\
& x^{L} \leq x^{k}+d \leq x^{U}
\end{aligned}
$$

where $P=\left[P_{1}, \ldots, P_{m}\right]$ is an $n \times m$ matrix, $\alpha_{1}^{T}=\left(\alpha_{1}, \ldots, \alpha_{m}\right)$ - m-vector.

- $\alpha^{T}$ denotes the transposition of the column vector $\alpha$.

- $|d|$ is the euclidean norm of vector $d \in R^{n}$.

- $m$ denotes the current number of trial points stored by the algorithm (equal to number of preserved linear approximations to the exact penalty functions).

The left-hand sides of inequalities (12) represents the approximations to the exact penalty function $e(x ; c)$ recalculated so that all of them are represented at the same point $x^{k}$ (the current approximate solution of the main penalty problem).

Formula $-\alpha_{i}+P_{i}^{T} d$ in (12) appears as the result of the construction of linear approximation of the minimized penalty function at the $\mathrm{i}$-th trial point $y_{i}$. All formulae (12) have been reformulated to describe the supporting hyperplanes directly by means of the current point $x^{k}$ and vector perpendicular to it (see Lemarechal (1977), Mifflin (1982), Kiwiel $(1985,1988))$.

Usually the dual problem to (11-14) is solved, e.g. Lemarechal (1977), Mifflin (1982), Kiwiel (1989). The reason is that one can easy generate the staring feasible point taking the optimal solution of the previous dual problem and assuming components corresponding to the newly added constraints equal to zero.

We have decided to use the primal one. The reasons are:

- We solve a transformed problem, that has a form of finding orthogonal projection onto a linearly constrained feasible set.

- The transformed problem is strictly convex.

- There exists an easy way to construct a starting feasible point for a new transformed direction finding problem on the basis of the optimal solution of the previous one (see sections 4 and 5 ).

- $Q, R$ factors of the matrix of active constraints at the optimal point of the previous problem after simple recalculation give the $Q, R$ factors of the matrix of constraints active at the starting point of the new transformed problem. 
The only difference between the consecutive problems (11-14) is that one constraint is added, eventually one deleted (it is always a constraint inactive at the optimal solution $\left(d^{k}, v^{k}\right)$ of the previous one and hence it may be neglected) and the linearizations are recalculated (it impacts exclusively the $\alpha_{i}^{k}$ scalars). The latter operation takes place only when the step is successfull. Then $x^{k+1}=x^{k}+d^{k}$ and we may take $d_{0}=0$ as the starting point for the QP problem number $(k+1)$. If the step is not successfull then the old linearizations do not vary and we take $d_{0}=d^{k}$ as the starting feasible point. The rules accepted when the stepsize differs from 1 are easily deduced in the same manner.

The only problem left is with the newly added constraint. Let the pair $\left(d^{k}, v^{k}\right)$ be the optimal solution of the $k$-th QP problem. Now two distinct cases are possible:

1) $\tilde{\alpha}_{p}+P_{p}^{T} d_{2} \leq v^{k}$

2) $\tilde{\alpha}_{p}+P_{p}^{T} d_{2}>v^{k}$.

In the first case, pair $\left(d_{0}, v^{k}\right)$ is the optimal solution of the problem number $(k+1)$. In the second one, much more interesting case, the newly added constraint will be surely active at the optimal solution of the new problem. This fact we use to eliminate variable $v$ and form the reduced primal direction search problem which is strictly convex while the original is merely convex.

\section{REDUCED DIRECTION SEARCH QP PROBLEM}

As a result of elimination of variable $v$ we obtain the following strictly convex QP program (least distance problem):

$$
\begin{aligned}
& \min _{d} \frac{1}{2}\left|d+P_{p}\right|^{2} \\
& \tilde{\alpha}_{i}-\alpha_{p}+\left(P_{i}-P_{p}\right)^{T} d \leq 0, \quad i=1, \ldots, m \\
& x^{L} \leq y^{k}+d \leq x^{k} \\
& y^{L} \leq A\left(y^{k}+d\right) \leq y^{U} .
\end{aligned}
$$

There exists many effective methods for solving such problems. We have chosen the Gill and Murray primal solution method Gill and Murray (1978). The details of the algorithm are omitted due to the lack of space. Gill and Murray's method requires a feasible starting point and maintains this property in all iterations.

In the second case discussed at the end of the previous section $\alpha_{p}+P_{p}^{T} d_{0}>v^{k}=$ $\alpha_{q}+P_{q}^{T} d_{0}$ hence in the new reduced problem we are substracting the value greater than $v^{k}$ which was substracted in the previous problem. Therefore $d_{0}$ shall be feasible for the new direction search problem.

Furthermore, point $d_{0}$ possesses some special properties as follows: 
i) set of active general linear and box constraints remains intact, i.e. is the same as it was in the optimal point $d^{k}$ of the previous reduced direction search subproblem.

ii) the set of active constraints reflecting the linear functions approximating from below the epigraph of the minimized nonsmooth function is not inherited. In fact, it may happen that none of these constraints shall be active at point $d_{0}$.

\section{REFERENCES}

Clarke, F.H. (1983) Optimization and nonsmooth Analysis. Wiley-Interscience, New York. Gill, P.E. and Murray, W. (1978) Numerically Stable Methods for Quadratic Programming. Mathematical Programming, 14, 349-72.

Kiwiel, K.C. (1985) Methods of descent for nondifferentiable optimization. Springer Verlag, Berlin, Heidelberg, New York.

Kiwiel, K.C. (1988) Some Computational Methods of Nondifferentiable Optimization. (in polish), Ossolineum, Wroclaw.

Kiwiel, K.C. (1989) A dual method for certain positive semidefinite quadratic programming problem. SIAM Journal on Scientific and Statistical Computing, 10, 175-86.

Lemarechal, C. (1977) Nonsmooth Optimization and Descent Methods. Technical Report RR-78-4, International Institute for Applied Systems Analysis, Laxenburg, Austria.

Mifflin, R. (1982) A Modification and an Extension of Lemarechal's Algorithm for Nonsmooth Minimization, in: Nondifferentiable and Variational Techniques in Optimization (eds. D.C. Sorensen and R.J.-B. Wets), Mathematical Programming Study 17, North Holland, Amsterdam. 